\title{
Folded MEMS Pyramid Inertial Measurement Unit
}

Sergei A. Zotov, Member, IEEE, Montgomery C. Rivers, Member, IEEE, Alexander A. Trusov, Member, IEEE, and Andrei M. Shkel, Senior Member, IEEE

\begin{abstract}
This paper reports a new approach for design and fabrication of chip-level inertial measurement units (IMUs). The method utilizes a 3-D foldable silicon-on-insulator (SOI) backbone with in-situ fabricated high-aspect-ratio sensors. A planar multisensor unit was fabricated and subsequently folded in a pyramidal shape, forming a compact IMU. Inertial characterization of the sensors integrated on the IMU pyramid was performed at atmospheric pressure. Structural rigidity and sensor axis alignment stability of the folded IMUs have been characterized under various environmental conditions, including vibration, thermal loading, thermal shock, and constant acceleration. The maximum angular misalignment due to variation in environmental conditions between IMU pyramid sidewalls was shown to be less than 4 and $0.2 \mathrm{mrad}$ for epoxy and solder reinforced structures, respectively. Vibration testing revealed no resonances up to $10 \mathrm{kHz}$ in the assembled 3-D structures. Our results confirm feasibility of the fabrication approach.
\end{abstract}

Index Terms-Inertial measurement unit (IMU), inertial sensors, microelectromechanical systems (MEMS), micromachined accelerometer, micromachined gyroscopes, 3-D MEMS.

\section{INTRODUCTION}

I NERTIAL sensing is used in a wide variety of applications including navigation, positioning, and targeting in defense, exploration, consumer electronics, automotive, and robotics industries [1], [2]. For complete detection of motion, rotation and linear acceleration must be measured along three nonplanar axes, requiring a six-axis Inertial Measurement Unit (IMU). Due to the increasing number of portable applications that require position and navigation information, miniaturization of IMUs is desirable. Besides development of high-performance inertial sensors, one major challenge in development of a compact IMU is combining multiple high-performance sensors capable of detecting rotational sand translational motion along independent sensitive axes with minimal mechanical and electrical parasitic cross-coupling. Overall, IMU performance directly depends on the rigidity of the supporting structure, to provide a stable alignment of the sensitive axes for a wide variety of environmental conditions [3].

Current methods for creating miniature IMUs typically rely on either assemblies of separate Printed Circuit Board (PCB) modules containing individual single-axis sensors [4], [5], or

Manuscript received February 01, 2011; revised May 16, 2011; accepted May 24, 2011. Date of publication June 27, 2011; date of current version October 21,2011 . This work was supported by the DARPA/MTO Grant W31P4Q-08-10008, program manager Dr. T. Akinwande. The associate editor coordinating the review of this paper and approving it for publication was Dr. Thomas Kenny.

The authors are with the University of California, Irvine, CA 92697 USA (e-mail: szotov@uci.edu; mrivers@uci.edu; atrusov@uci.edu; ashkel@uci.edu).

Color versions of one or more of the figures in this paper are available online at http://ieeexplore.ieee.org.

Digital Object Identifier 10.1109/JSEN.2011.2160719



Fig. 1. A prototype of the folded MEMS pyramid IMU with co-fabricated inertial sensors, electrical interconnects on flexible hinges, and sensor encapsulation lids.

consist of multi-axis sensors which utilize multiple degrees of freedom of a single proof mass [6], [7]. While the PCB-level approach allows for the use of optimized, high-performance single-axis sensors, its further miniaturization is limited. In contrast, integration of multi-axis sensors on the same substrate has a potential to provide a small form factor IMU, but suffers from more complex fabrication. This approach typically involves compromises in sensor design and performance, and presents a major difficulty in decoupling signals between the sensitive axes. The choice between existing approaches leads to a tradeoff between size and performance, leaving no clear path toward a high-performance chip-level IMU.

In this paper, we report development of a novel approach for design and fabrication of rigid 3-D IMU structures that may overcome the undesirable tradeoffs that exist with current approaches. An introduction of the folded MEMS fabrication approach is given in Section II, followed by a description of the fabrication process in Section III. Section IV presents results of experimental characterization of structural rigidity as well as angular misalignment between IMU pyramid sidewalls. Section V describes inertial characterization of gyroscopes and accelerometers fabricated on a pyramidal folded MEMS IMU. Section VI concludes this paper with a summary and discussion of the results.

\section{FOLDED MEMS FABRICATION APPROACH}

In the proposed approach, single-axis sensors are implemented on a flat surface of a wafer, and then subsequently folded in a 3-D spatial configuration, thus forming a compact IMU [8]-[10]. Fig. 1 shows a fabricated prototype of an IMU-pyramid structure with inertial sensors located on the 


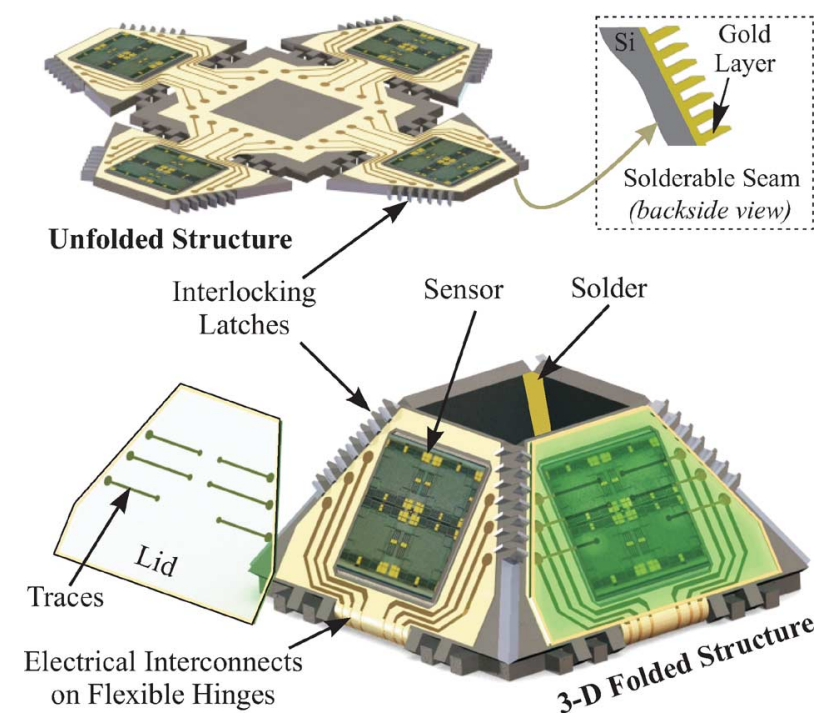

Fig. 2. Design features of a folded MEMS pyramid IMU including interlocking latches, SOI sensors, flexible interconnect hinges, and packaging lids.

sidewalls. Sensors are fabricated in parallel with a foldable backbone and after assembly, the process results in a complete IMU microsystem with a package volume less than $0.5 \mathrm{~cm}^{3}$. The IMU architecture utilizes flexible hinges that allow the dies to fold together and lock in place. The interlocking latches are located along the edges of each sidewall and are fabricated as a part of the fabrication sequence. Electrical interconnects transmit signals from each sidewall to the base of the structure, and serve as an interface between sensors and external signal conditioning circuitry. With all bond pads located on the bottom, flip-chip attachment is used to mount the folded MEMS IMU to a PCB.

Even though the proposed approach has many advantages, several design challenges should be considered, including the structural rigidity of the folded microsystem, durability, sensor alignment, and packaging. Design of the IMU involves a number of elements, Fig. 2, including a rigid backbone, inertial sensors, mechanical latches, flexible hinges, electrical interconnects, and glass lids for packaging.

Structurally, the backbone is comprised of a number of silicon panels, each containing one or several inertial sensors. Due to its high rigidity and long-term mechanical performance [11], silicon is a suitable material for an inertial sensor platform. The ratio of the Young's modulus to the material density is also much larger than that of most materials. This property allows for a lightweight IMU with high rigidity, and minimizes the forces induced on sidewalls from environmental loading, such as shock, constant acceleration, and vibration. Additionally, multiple fabrication techniques exist for micromachining silicon and are readily available in most foundries, making silicon an optimal choice for the IMU structures.

An interlocking latch design is used along each edge that provides initial rigidity after assembly and also ensures proper sensors alignment during assembly of the folded structure [10]. Gold is deposited on the backside of the latches to allow for internal structural reinforcement using solder. By utilizing a metallic bond at the cross-latched interfaces, a solid joint is made between sidewalls to provide permanent rigidity and stable alignment of inertial sensor axes on the 3-D IMU structure.

Compliant hinges are an integral part of the fabrication sequence. Significant flexibility of hinges is required for folding the sidewalls into place, as well as durability, the capability of carrying electrical signals, and good adhesion to silicon and metal. Polyimide is identified as a candidate material exhibiting the required properties. To minimize stress induced in the polyimide after assembly, hinges are designed with $1100 \mu \mathrm{m}$ spacing between the panels, allowing for a required bending radius of $500 \mu \mathrm{m}$. Electrical interconnects are integrated onto the polyimide substrate, allowing the transmission of signals from each sensor on the side panels to the base of the pyramid for subsequent flip-chip attachment to a PCB.

Accelerometers and gyroscopes are located on the sidewalls of the MEMS IMU structures, and are fabricated using the same process used for creating the silicon backbone, hinges, and latches. Utilizing high-aspect-ratio etching for sensor fabrication, high-performance devices can be implemented on the sidewalls of the 3-D MEMS IMU structures. For optimal performance, inertial sensors generally require hermetic sealing or vacuum packaging to reduce damping and provide environmental protection. As a first step toward this goal, we implement and demonstrate lid designs for dust protection. In this application, the glass encapsulation lids have been designed, fabricated, and demonstrated as a part of the MEMS IMU structures. In the proposed method, the glass lids include electrical interconnects to function as a substitute for wirebonding of individual sensors. Each lid is designed with bond pads that directly correspond to the sensor anchors as well as the bond pads located on the polyimide substrate. The developed packaging sequence is potentially compatible with a glass lid vacuum sealing process. Development of the vacuum sealing process, however, is outside the scope of this paper.

\section{FABRICATION AND PACKAGING}

\section{A. Process Overview}

Fabrication of the folded IMU structures involves creating the sensors, patterning polyimide around the sensors to function as hinges, and deposition of metal interconnects on top of the polyimide, Fig. 3. Assembly is accomplished by folding the panels and locking them in place, utilizing the interlocking latches. When implementing the six-axis IMU, a cube is the structure which would require a minimal coordinate transformation to obtain 6-DOF position and orientation data. A pyramidic structure can also in principle detect 6-DOF acceleration and orientation data. Although this requires more complex signal processing, a pyramid has structural advantages compared to a cube. A pyramid is mechanically more rigid than a cube, and therefore provides a preferred platform for improvement of structural rigidity. Folded 3-D IMU pyramids and cubes have been explored [10], and other types of IMU structures are feasible for implementation utilizing the same approach. A fabrication process, Fig. 4, consisting of eight fabrication steps has been developed for manufacturing folded MEMS IMU prototypes. For simplification purposes, the complete fabrication pro- 


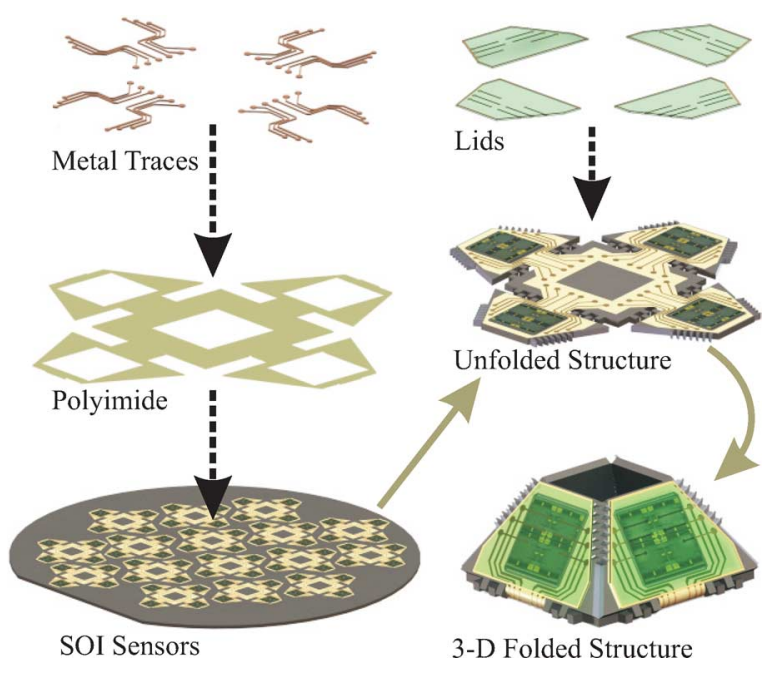

Fig. 3. Fabrication approach for 3-D folded MEMS IMU structures.

cedure is represented in the figure as five general steps with the details of each explained in this section.

\section{B. Flexible Hinges}

The first step of the fabrication process is forming the flexible hinges, Fig. 4(a). Polyimide is deposited onto the substrate by spin-coating. For durability, it is desired to have a thick layer of polyimide capable of withstanding dynamic loading over an extended period of time. The polyimide used in this process is HD-4110, which has a nominal thickness of approximately $20 \mu \mathrm{m}$ after curing. This type of polyimide contains a negative photosensitive resist to allow for direct lithographic patterning. The fabrication process assures well-maintained adhesion of polyimide to the silicon substrate as well as to the metal interconnects.

The deposition recipe parameters have been tailored for maximum adhesion properties, which includes optimization of cleaning conditions, material deposition, and baking profile. Spin-coating is performed at a rate of $1500 \mathrm{rpm}$ for $40 \mathrm{~s}$. Solvents are then removed from the liquid-state polyimide by baking on a hot plate at $120^{\circ} \mathrm{C}$ for $12 \mathrm{~min}$. The adhesion layer begins to form between the polyimide and silicon after solvents are removed. Patterning is done by exposing the polyimide to I-line ultraviolet (UV) light with an intensity of $10 \mathrm{~mJ} / \mathrm{cm}^{2}$ for a duration of $60 \mathrm{~s}$. A postexposure bake (PEB) is then performed by heating the wafer on a hotplate at $120{ }^{\circ} \mathrm{C}$ for $5 \mathrm{~min}$. This provides additional adhesion of the polyimide to the silicon substrate and also increases etch selectivity during development.

To fully cure the polyimide, a ramp-and-hold recipe is performed in a continuously purged nitrogen environment. Initially, the temperature is increased from $25^{\circ} \mathrm{C}$ to $200{ }^{\circ} \mathrm{C}$ at a rate of $8{ }^{\circ} \mathrm{C}$ per minute. The temperature is then held at $200{ }^{\circ} \mathrm{C}$ for $30 \mathrm{~min}$, followed by ramping the temperature up to $375^{\circ} \mathrm{C}$ at $8{ }^{\circ} \mathrm{C}$ per minute. After $1 \mathrm{~h}$ at $375^{\circ} \mathrm{C}$, the polyimide is completely cured, and the temperature is then decreased to $25^{\circ} \mathrm{C}$ over a period of several hours. Once cured, the final thickness of polyimide is $18 \mu \mathrm{m}$. Using this recipe, the polyimide substrate

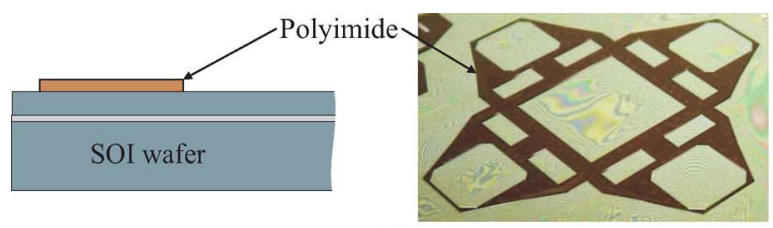

(a)

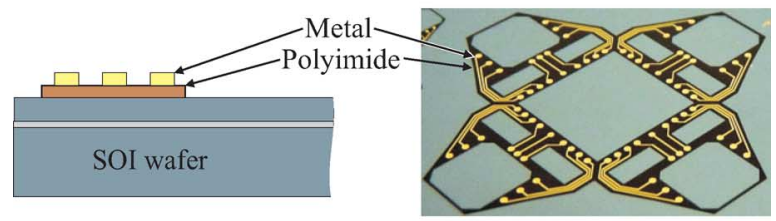

(b)

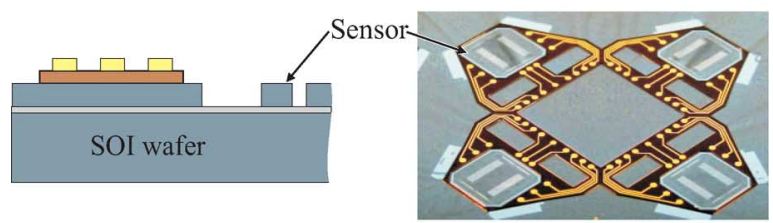

(c)

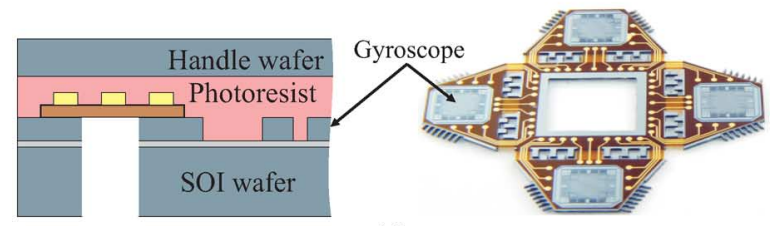

(d)

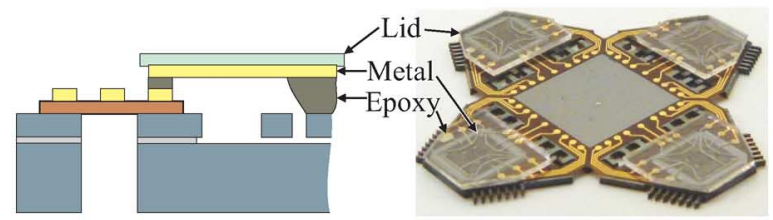

(e)

Fig. 4. Fabrication process for folded IMU structures. The process includes fabrication of integrated sensors, polyimide hinges, gold interconnects, and placement of separately fabricated glass lids. (a) Polyimide deposition and patterning. (b) Metal deposition and patterning. (c) Definition of sensors using photolithography and DRIE. (d) Backside through-etch using DRIE and release of foldable structures. (e) Align and place glass lids to each sidewall.

maintains adhesion to both silicon and metal throughout the entire fabrication process and provides a durable flexible substrate to function as hinges for folded MEMS IMU structures.

\section{Electrical Interconnects}

Electrical interconnects are deposited on top of the cured polyimide and patterned using a liftoff technique, Fig. 4(b). For this process, $\mathrm{AZ} \mathrm{nLoF} 2035$ photoresist is used as the masking material, which is specifically designed for metal liftoff patterning. Physical vapor deposition (PVD) is performed using electron beam evaporation to create the desired metal layers. Initially, a $500 \AA$ adhesion layer of chrome is deposited, followed by $5000 \AA$ of gold. Impedance of traces is minimal with this procedure, creating interconnects with a maximum measured resistance of $3.8 \Omega$.

\section{Silicon-on-Insulator (SOI) Inertial Sensors}

Sensors are made using silicon-on-insulator (SOI) wafers, enabling high-aspect-ratio device features for optimal per- 
formance. By utilizing SOI fabrication, there is a significant flexibility in the type of inertial sensor designs that can be implemented.

In this feasibility study, the sensors are defined using a photoresist mask with a minimum feature size of $8 \mu \mathrm{m}$. High-aspect-ratio etching is performed using Deep Reactive Ion Etching (DRIE) to achieve an etch depth of $50 \mu \mathrm{m}$, exposing the buried silicon dioxide layer, Fig. 4(c). By utilizing the entire thickness of the device layer, inertial sensor performance is optimized, and can be further improved by increasing the thickness of the device layer from 50 to $100-200 \mu \mathrm{m}$, in tandem with increasing of the aspect ratio of etching. After defining the sensor features, fabrication of the top substrate is finished. Before the MEMS structures are released, backside etching of the handle wafer must be completed. Due to the fragility of sensor features, the device layer is protected by a blanket coating of AZ P4620 photoresist, followed by applying a layer of low-tack dicing tape. After the remaining fabrication processes are complete, these protective materials are removed by immersing in acetone.

\section{E. Singulation of Foldable Structures}

Singulation of the structures is done by selectively etching through the wafer from the backside, enabling the ability to fold the flat structure into an IMU. This process requires etching a total of $550 \mu \mathrm{m}$ of silicon, as well as $5 \mu \mathrm{m}$ of silicon dioxide, separating $500 \mu \mathrm{m}$ of the SOI handle wafer and $50 \mu \mathrm{m}$ of the device layer. A mask, matching the selective etching rate of silicon and photoresist, is created by patterning a double-layer of AZ P4620 photoresist to achieve a thickness of $28 \mu \mathrm{m}$. A handle wafer is then attached to the opposite side of the device wafer using a layer of photoresist, Fig. 4(e). Backside etching then ensues using DRIE until the handle wafer is completely etched in the defined trenches. Removal of the buried oxide layer is performed with buffered oxide etch (BOE) because it does not affect mask adhesion, nor does it etch the photoresist. Once the oxide layer is fully etched, backside processing continues using DRIE to etch through the remaining $50 \mu \mathrm{m}$ of silicon. After the backside etch is complete, the sample is immersed in acetone to dissolve the photoresist between the handle wafer and manufactured device wafer and the unassembled IMUs are extracted. The foldable structures are removed from the wafer and cleaned to prepare the sensors for release. Acetone is initially used to dissolve the majority of the existing residue, followed by dry removal with oxygen plasma using the following chamber conditions: $200 \mathrm{~W}$ coil power and a pressure of 200 mTorr.

\section{F. SOI Sensor Release}

The next step in the fabrication process is to release the SOI sensors from the substrate. Etching is performed using either $20 \%$ hydrofluoric acid (HF) or 6:1 Buffered Oxide Etch (BOE), Fig. 4(d). Stiction of the sensor proof masses to the underlying substrate due to capillary drying effects is prevented by immersing the structures in a bath of isopropanol heated to $50{ }^{\circ} \mathrm{C}$ for a duration of $5 \mathrm{~min}$. The structures are then dried at the same elevated temperature to ensure rapid evaporation. Because the surface tension of isopropanol is very low, capillary drying effects are eliminated. After successfully releasing all sensors, fabrication of the folded MEMS IMU structures is complete and the devices are ready for packaging.

\section{G. Lid Fabrication}

Glass lids have been designed and fabricated for the purpose of protecting the inertial sensors from the environment, as well as providing electrical connections to the sensor anchors. For complete packaging of the folded MEMS IMU pyramid, individual sensors are first connected to the electrical traces, followed by attachment of the overall structure to a PCB or other suitable interface, such as a DIP package. Sensor anchors are connected to the electrical traces on the polyimide substrate using glass encapsulation lids containing metal traces. Waferlevel fabrication of the lids is performed on a $500 \mu \mathrm{m}$ D-263 glass substrate and metal is patterned using a liftoff process to define the electrical interconnects, Fig. 2. Deposition of metal is done utilizing the same procedure as for the IMU structure interconnects to create a $5000 \AA$ layer of gold atop a $500 \AA$ adhesion layer of chrome. After fabrication, the wafer is diced to separate each individual encapsulation lid. Impedance of the traces manufactured with this procedure is measured to have a maximum resistance of less than $1 \Omega$, which is adequate for implementation of the folded IMU devices.

\section{H. Lid Attachment}

For stable long-term operation, inertial sensors must be protected from the environment. The glass interconnect lids can be used not only as an effective replacement for wirebonding, but also can be implemented as a hermetic sealing mechanism. Additionally, the lids are suitable for vacuum sealing by using eutectic solder to bond the gold-plated border to the sensor package, which will minimize damping and significantly improve overall sensor performance. Initial packaging in this work, however, is done without sealing the devices and characterization is performed at ambient pressure to prove feasibility of the approach.

In this feasibility study, bonding of the interconnect lids is done utilizing a WestBond 7201CR-79 die bonder. Silver-filled conductive epoxy (Ablebond 84-1LMI) is used to create the electrical connections between the sensor anchors and the bond pads on the lids. Epoxy is precisely placed onto all sensor anchors and bond pads located on polyimide. The glass lids are then aligned and placed above the sensors using flip-chip bonding to create the electrical connections. Because the polyimide layer is approximately $20 \mu \mathrm{m}$ in height, the glass lids rest above the sensors without causing overflow of the epoxy onto the sensor features. Curing of the conductive epoxy is performed after all lids are aligned by heating the structure at $150{ }^{\circ} \mathrm{C}$ for $1 \mathrm{~h}$. After curing, the solvents are evaporated from the silver-filled epoxy, leaving a permanent conductive medium between the folded IMU structure bond pads and the encapsulation lids.

\section{Assembly and Reinforcement}

After the lids are attached to all sensors and the conductive epoxy is cured, the panels are folded into the pyramidal shape. Currently, structures are assembled manually using a magnifying lens and small hand held tools to fold the sidewalls 


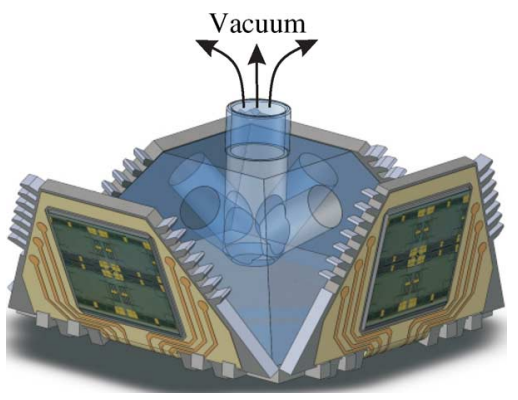

Fig. 5. Assembly of IMU pyramid using a molded plastic insert with vacuum channels used to assemble the folded structures.

together. However, a method for precision assembly using a molded insert to act as a vacuum-substrate jig for assembly has been explored, Fig. 5. Because the entire structure is manufactured on the wafer-level, all structural elements are lithographically defined to a tolerance on the order of a few microns. The largest imperfection is induced during the backside DRIE process, which causes a variation of approximately $20 \mu \mathrm{m}$ for a $550 \mu \mathrm{m}$ etch depth. For each sidewall, designed $11 \mathrm{~mm}$ from edge to edge, this will create a total angular variation from the designed alignment of $3.6 \mathrm{mrad}$.

After assembly is complete, the IMU structure needs to be reinforced to maintain the original alignment of sensors. In our study, we explored epoxy and eutectic soldering reinforcement at the sidewall interfaces. Epoxy reinforcement is achieved by applying adhesive to the latches after assembly is complete, followed by curing to create a rigid interface between each sidewall. Soldering has also been explored to provide metallic bonds between folded sidewalls for further rigidity enhancement.

Because silicon does not inherently allow for wetting of solder, a layer of gold is first deposited onto the bonding joints, Fig. 2. Metal deposition is conducted in post-fabrication processing by mounting the unfolded structure facedown, leaving the backside exposed. With the current process, the entire backside area is coated, however, a shadow mask or lithography can also be used to mask the sidewalls and selectively deposit metal only on the latches. Similar to metal deposition processes described above, electron beam evaporation is employed to create a $500 \AA$ adhesion layer of chrome followed by $5000 \AA$ of gold on the backside of the interlocking latches. Solder paste is then applied to the metalized latches before assembly and reflown after folding the sidewalls in place by heating the structure above the $183{ }^{\circ} \mathrm{C}$ solder melting point.

\section{FoldED IMU STRUCTURAL CHARACTERIZATION}

\section{A. Overview}

Assuming the challenges with design of high-performance sensors are solved, a major challenge with creating high-performance IMUs is to maintain the original sensor alignment under various environmental conditions [3], [12], [13]. Angular deflections and flexure of the 3-D folded IMU structure causes bias errors in the output signals. If permanent misalignment occurs, the IMU must be recalibrated to correct for these errors. However, this is largely unacceptable for most applications. Using the folded MEMS approach, interlocking latches along



(a)

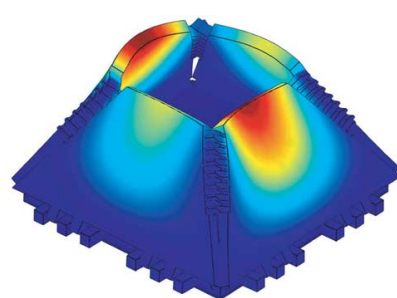

(b)
Fig. 6. Finite-element modeling showing the first two modalfrequencies of an IMU pyramid reinforced with eutectic solder. (a) First mode at $63 \mathrm{kHz}$. (b) Second mode at $70 \mathrm{kHz}$.

the edges of each sidewall provide initial rigidity after assembly, and also assist in properly aligning the sidewalls. More applications, however, require additional reinforcement. In this paper, we explore rigidity enhancement utilizing solder and epoxy. Environmental tests performed on the IMU pyramids consist of vibration, temperature, temperature shock, and constant acceleration response.

\section{B. Vibration Testing}

Modal frequencies of a solder-reinforced IMU pyramid is predicted using Finite-Element Analysis (FEA) of a 3-D model using COMSOL FEA software. An eigenfrequency analysis of the folded pyramid structure is conducted. In this analysis, the complete geometry is modeled using a static mesh with 154132 tetrahedral elements. The model consists of four independent sidewalls and a baseplate. Each panel has a thickness of $550 \mu \mathrm{m}$. The footprint of the baseplate is $11 \times 11 \mathrm{~mm}$, and the opening at the top of the folded structure is $5 \times 5 \mathrm{~mm}$. The angle between each sidewall and the bottom panel is $58.9^{\circ}$. All structural panels are defined as crystalline silicon with an isotropic modulus of elasticity of $170 \mathrm{GPa}$. To model soldered connections at the interfaces between silicon panels, the bonded regions are defined to have a modulus of elasticity of $50 \mathrm{GPa}$. The sidewalls are also bonded to the baseplate along each edge, using a thin seam of solder with a $500 \mu \mathrm{m}$ diameter. Geometry is fixed using a $100 \mu \mathrm{m}$ solder layer beneath the bottom of the structure to represent attachment of the pyramid to an external package. Results indicate the first resonant mode to be approximately $63 \mathrm{kHz}$, in which each adjacent pair of sidewalls shows antisymmetric flexing, Fig. 6(a). The second modal frequency occurs at $70 \mathrm{kHz}$ and depicts a mode shape with symmetric flexing of adjacent sidewalls, Fig. 6(b). For most IMU applications, environmental vibrations occur up to approximately $10 \mathrm{kHz}$, therefore the simulation confirms that frequencies of the first two modes will not be excited during normal operation.

Using a SPEKTRA SE-09 vertical shaker, sinusoidal vibrations from 20 to $50000 \mathrm{~Hz}$ is applied to the folded IMU structures. A Polytec laser vibrometer is used to measure the response of the structures throughout the sweep. Vibration data is collected at both the top of the sidewalls and also at the base of the structure. By comparing the difference between the two sets of data, response of the sidewall is calculated and effects from the mounting setup are eliminated.

Measured responses reveal no resonances in the assembled 3-D structures up to $10 \mathrm{kHz}$, Fig. 7. First resonant modes are 

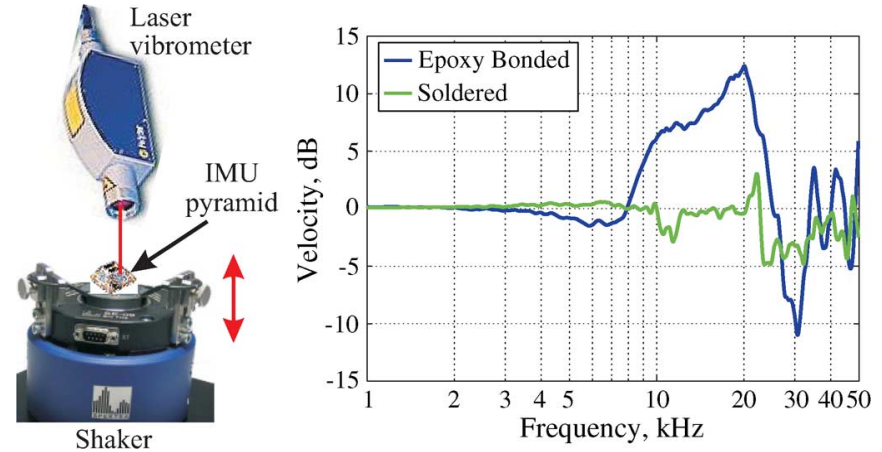

Fig. 7. Measured frequency responses of a 3-D folded IMU pyramid responses reveal no resonances peaks up to $10 \mathrm{kHz}$.

observed above $10 \mathrm{kHz}$ in the epoxy-bonded pyramids, which is suitable for most IMU applications. In contrast, the soldered 3-D IMUs do not exhibit any significant resonant modes up to $50 \mathrm{kHz}$, suggesting suitability for very harsh vibrational environments. These results indicate an increase in structural stiffness for soldered pyramids versus epoxy-bonded pyramids. Overall, feasibility of both reinforcement methods is proven, with solder providing superior rigidity for the 3-D folded structures.

\section{Evaluation of Sensor Alignment}

Initial alignment of sensors after assembly and packaging are designed to have well-defined sense axes in their relative coordinate frame. However, due to fabrication and assembly imperfections, calibration is necessary to fully characterize the complete IMU. Once calibrated, slight misalignments of the sensors will cause errors in inertial signal detection. Therefore, performance of an IMU directly depends on the ability of the supporting architecture to rigidly maintain the initial sensor alignment. The folded structures must be robust to environmental conditions such as thermal changes, vibration, acceleration loading, and shock. Characterization of structural rigidity is performed by measuring misalignment of the IMU sidewalls before and after various environmental loads are applied. A comparison is made between epoxy bonding and eutectic soldering to determine the performance of each reinforcement technique in varying environmental conditions.

An optical characterization method was developed to precisely measure the relative misalignments of the pyramid sidewalls, Fig. 8. Folded 3-D structures are rotated using a precision-controlled dual-axis Ideal Aerosmith 2102 rate table with an angular accuracy of $0.15 \mathrm{mrad}$ and repeatability of $0.05 \mathrm{mrad}$. At the same time, reflection of a collimated laser beam from the pyramid sidewalls is observed using a calibrated detection screen at a $1.5 \mathrm{~m}$ distance. The following procedure is used to obtain misalignment data from each sidewall before and after each environmental condition is applied.

1) Mark the position of the laser beam reflected off of sidewall. This acts as a reference for later measurements.

2) Precisely rotate the pyramid by $90^{\circ}$ and mark the position of the laser beam reflected off of sidewall 2 .

3) Repeat Step 2 for sidewalls 3 and 4.

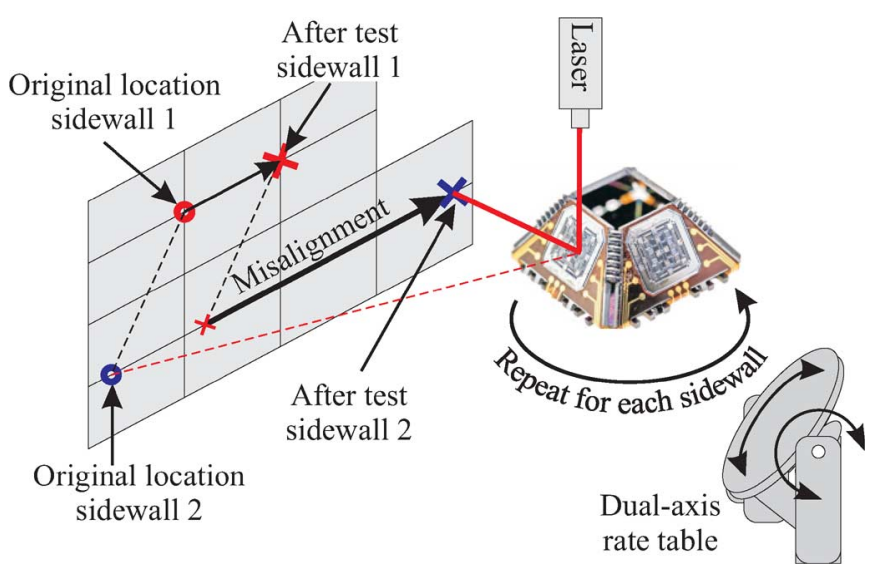

Fig. 8. Optical measurement setup for alignment characterization of folded IMU sensor axes.

4) Remove the IMU pyramid and conduct environmental testing.

5) Place the IMU pyramid back onto the test stage and adjust the rate table such that the sidewall 1 beam location is very near the original reference mark.

6) Mark the new position of the laser beam reflected off of sidewall 1 as the second reference.

7) Measure the distance between the two reference marks from sidewall 1.

8) Precisely rotate the pyramid by $90^{\circ}$ and mark the position of laser beam reflected off of sidewall 2 .

9) Measure the distance between the respective two marks for sidewall 2.

10) Subtract the sidewall 1 distance from the sidewall 2 distance and multiply by the calibrated sensitivity of the detection grid to calculate misalignment.

11) Repeat Steps 8, 9, and 10 for sidewalls 3 and 4.

1) Elevated Temperature: For static thermal testing, IMU pyramid structures are placed on a miniature heater located on the dual-axis rate table. Prior to heating, the initial angular position of each sidewall is measured. Temperature of the pyramid is then elevated to $85^{\circ} \mathrm{C}$ over a period of $25 \mathrm{~min}$. Temperature is held at $85^{\circ} \mathrm{C}$ for $25 \mathrm{~min}$ to allow for steady-state conditions. Misalignment data from each sidewall is extracted at the elevated temperature using the above procedure, yielding a maximum variance of $1.7 \mathrm{mrad}$ for an epoxy-bonded IMU pyramid.

2) Temperature Cycles: Misalignment of the IMU pyramid structures is measured after thermally cycling from room temperature to $-40{ }^{\circ} \mathrm{C}$ and $85^{\circ} \mathrm{C}$, respectively. Before testing, angular position of the sidewalls is measured. The IMU structure is then mounted inside a thermal chamber (TestEquity model 107) and heated to $85{ }^{\circ} \mathrm{C}$. After holding the temperature for $25 \mathrm{~min}$, the IMU is gradually cooled to room temperature. Once steady-state temperature of the folded structure is reached, detection of misalignment is conducted to determine the effects of the process. The same process was performed and the corresponding misalignment data was obtained after thermally cycling the IMU from room temperature to $-40{ }^{\circ} \mathrm{C}$. Results indicate a maximum angular sidewall variance for an epoxy-bonded IMU structure of 1.6 and $3.0 \mathrm{mrad}$ for heating and cooling cycles, respectively. 
TABLE I

Measured ANGUlar MisalignMENT OF Folded MEMS IMU SENSOR AXeS FOR EPOXY AND SOLDER REINFORCEMENT METHODS

\begin{tabular}{|c|c|c|}
\hline \multirow[t]{2}{*}{$\begin{array}{l}\text { Environmental Test } \\
\text { (vs. initial position at } 25^{\circ} \mathrm{C} \text { ) }\end{array}$} & \multicolumn{2}{|c|}{$\begin{array}{l}\text { Max. Misalignment } \\
(\mathrm{mrad})\end{array}$} \\
\hline & Eроху & Solder \\
\hline $\begin{array}{l}25^{\circ} \mathrm{C} \rightarrow 85^{\circ} \mathrm{C} \\
\text { elevated temperature }\end{array}$ & $1.7 \pm 0.2$ & $<0.2^{*}$ \\
\hline $\begin{array}{l}25^{\circ} \mathrm{C} \rightarrow 85^{\circ} \mathrm{C} \rightarrow 25^{\circ} \mathrm{C} \\
\text { thermal cycle, } 25 \mathrm{~min}\end{array}$ & $1.6 \pm 0.2$ & $<0.2$ \\
\hline $\begin{array}{l}25^{\circ} \mathrm{C} \rightarrow-40{ }^{\circ} \mathrm{C} \rightarrow 25^{\circ} \mathrm{C} \\
\text { thermal cycle, } 25 \mathrm{~min}\end{array}$ & $3.0 \pm 0.2$ & $<0.2$ \\
\hline $\begin{array}{l}25^{\circ} \mathrm{C} \rightarrow 85^{\circ} \mathrm{C} \rightarrow 25^{\circ} \mathrm{C} \\
\text { thermal shock, } 3 \mathrm{~s}, 3 \text { cycles }\end{array}$ & $3.0 \pm 0.2$ & $<0.2$ \\
\hline $\begin{array}{l}25^{\circ} \mathrm{C} \rightarrow-40{ }^{\circ} \mathrm{C} \rightarrow 25^{\circ} \mathrm{C} \\
\text { thermal shock, } 3 \mathrm{~s}, 3 \text { cycles }\end{array}$ & $3.5 \pm 0.2$ & $<0.2$ \\
\hline $\begin{array}{l}\text { Constant acceleration } \\
60 \mathrm{~g} \text { for } 1 \mathrm{~min} \text { for each axis }\end{array}$ & $1.8 \pm 0.2$ & $<0.2$ \\
\hline
\end{tabular}

3) Temperature Shock: Thermal shock is also performed on the folded IMU pyramids, both for cold and hot temperatures. After measuring the initial angular position of each sidewall, the structures are transferred into an $85^{\circ} \mathrm{C}$ environment with a transition period of approximately $3 \mathrm{~s}$. Temperature is maintained for $25 \mathrm{~min}$ to ensure the entire structure is at steady-state. The pyramid is then transferred to room temperature within approximately $3 \mathrm{~s}$ to apply a negative thermal shock. Utilizing the same procedure, folded IMU pyramids are exposed to thermal shock from room temperature to $-40{ }^{\circ} \mathrm{C}$, then back to room temperature. Each experiment is repeated three times consecutively for each the hot and cold temperature tests to determine the effects of multiple cycles. Misalignment data from each sidewall on an epoxy-bonded pyramid results in a maximum variance of 3.0 and $3.5 \mathrm{mrad}$ for the $85^{\circ} \mathrm{C}$ and $-40{ }^{\circ} \mathrm{C}$ thermal experiments, respectively.

4) Constant Acceleration: For applications in which acceleration loads are constantly applied, folded MEMS IMU structures must withstand the forces involved. To determine suitability for such purposes, constant acceleration is applied to IMU pyramids by rotating the devices on a centrifuge with a known rotation rate. Loading is applied to the folded structure along three independent axes by mounting the IMU at orthogonal angles for each experiment. The setup is capable of providing up to $60 \mathrm{~g}$ of acceleration on the test sample. Misalignment of the sidewalls is measured before and after the experiment, yielding a maximum angular variance of $1.8 \mathrm{mrad}$ between sidewalls for an epoxy-bonded IMU structure.

Results of all alignment stability tests comparing epoxy and solder-bonded IMUs are shown in Table I. Structures reinforced with epoxy show a maximum alignment drift of $3.5 \mathrm{mrad}$ or less under each applied environmental condition. In contrast, the soldered structures provide stability better than $0.2 \mathrm{mrad}$. The lower limit of detection of the measurement setup is approximately the same, therefore the misalignment of the solder-bonded structures is likely much lower than the measured data. These results indicate feasibility of reinforced 3-D folded MEMS structures for a wide variety of environmentally robust IMU applications.



Fig. 9. SEM image of a MEMS accelerometer fabricated on the pyramid IMU sidewall.

5) Mechanical Shock: Utilizing the FEA model described above, shock loading is simulated for the pyramidic IMU structures. Shock is applied using gun-hard parameters with an amplitude of $30000 \mathrm{~g}$ over an interval of $7 \mathrm{~ms}$ [20]. Applying these parameters to the model along each $\mathrm{X}, \mathrm{Y}$, and $\mathrm{Z}$ directions yields a maximum misalignment of $0.5 \mathrm{mrad}$ and stress of $200 \mathrm{MPa}$. Comparing the stress induced to the $7000 \mathrm{MPa}$ yield strength of silicon, the structure will not fail under gun-hard conditions. For most applications, the slight misalignment produced during the simulation will not adversely affect the IMU data. Shock was also applied experimentally using an impact bar and a striker, with the pyramid under test mounted to the end of the bar. A Polytec laser vibrometer was focused onto the top of the pyramid to measure the acceleration induced into the structure. Tests show that the structures are capable of surviving up to 389 $\mathrm{g}$ of acceleration over a $0.5 \mathrm{~ms}$ time interval. It is believed that the structures will survive much higher values of acceleration, however the test setup is only capable of approximately $400 \mathrm{~g}$.

\section{INERTIAL SENSOR CHARACTERIZATION}

This section reports experimental characterization of accelerometers and gyroscopes fabricated by the process described in Section III. The presented results illustrate the feasibility of the process to produce functional devices.

\section{A. Accelerometer Characterization}

Design of the IMU accelerometers consist of a proof mass suspended by folded springs attached to anchors, parallel plate sense electrodes, and electrostatic comb drives, Fig. 9. Resonant detection is utilized due to its inherent high dynamic range, quasi-digital output [14]-[16], and resilience to parasitic capacitance noise.

Accelerometer performance is tested by measuring response to gravity at predefined angles using a tilt stage. The proof mass of the accelerometer is excited at a resonant frequency of $1.1 \mathrm{kHz}$ using a combination of DC and AC voltage applied to the anchored drive-mode electrodes. A variable DC tuning voltage is applied to one of the opposing banks of parallel-plate sense electrodes to provide an initial displacement of the proof mass. This modifies the original capacitive gap, and thus the overall sensitivity is changed [16], [17]. A carrier signal is applied to the proof mass to allow for frequency modulation detection using a lock-in amplifier, Fig. 10. The carrier frequency 




Fig. 10. Signal detection schematic used for accelerometer characterization.



Fig. 11. Measured acceleration response of a resonant accelerometer integrated on folded IMU pyramid for $20 \mathrm{~V}$ and $30 \mathrm{~V}$ tuning voltage. Inset: Optical photograph of an IMU pyramid sidewall with a resonant accelerometer.

utilized is at $52 \mathrm{kHz}$, which is much greater than the operational frequency of the accelerometer and thus has negligible effects on the proof mass motion. The sensor output signal is amplified using a Trans-Impedance Amplifier (TIA), followed by demodulation of the carrier frequency. Vertical angles are applied in increments of $5^{\circ}$ to induce acceleration ranging from $0-1 \mathrm{~g}$. At each position, the resonant frequency is measured to determine its shift caused by the input acceleration, with the results plotted in Fig. 11. Using a DC bias voltage of $20 \mathrm{~V}$ applied to the tuning electrodes, the sensitivity is determined to be $1.8 \mathrm{~Hz} / \mathrm{g}$. When $30 \mathrm{~V}$ bias is used, the scale factor is increased to $3.7 \mathrm{~Hz} / \mathrm{g}$.

\section{B. Gyroscope Characterization}

Architecture of the gyroscope consists of differential drive and sense electrodes and a large 2-DOF proof mass supported with folded suspension beams, Fig. 12. Angular rate performance of a gyroscope fabricated on an IMU pyramid sidewall is experimentally characterized in air. Rotation rate response is tested by mounting the structure to a computer-controlled Ideal Aerosmith 1291BR rate table. The drive mode of the gyroscope is excited into resonance at $1.6 \mathrm{kHz}$ using a combination of 30 VDC bias and 5 VAC driving signal applied to the anchored drive-mode electrodes. Separation of the useful signal from the feed-through signal is accomplished using Electromechanical

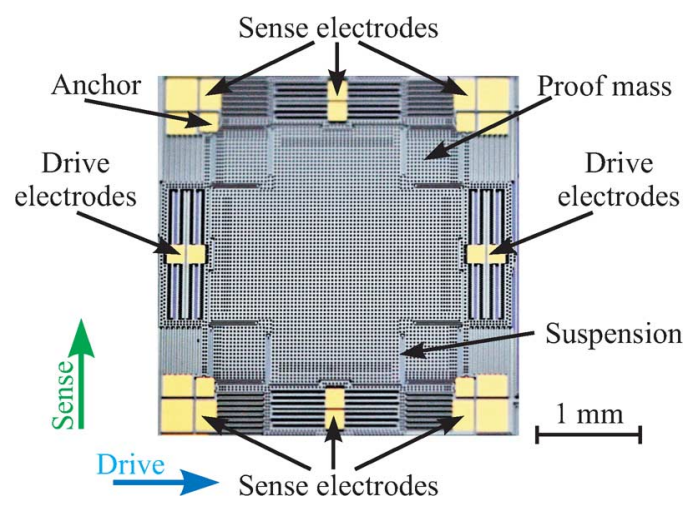

Fig. 12. Optical photograph of a MEMS gyroscope integrated on the pyramid IMU sidewalls.

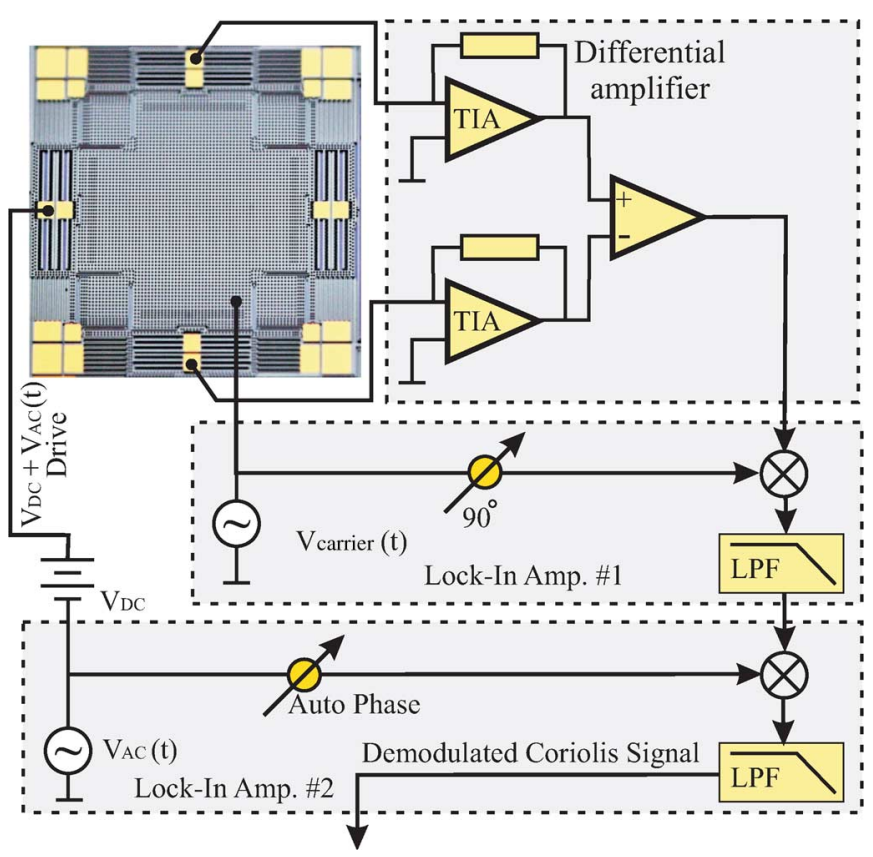

Fig. 13. Drive and detection schematic for gyroscope characterization.

Amplitude Modulation (EAM), where a carrier voltage of $3.5 \mathrm{Vrms}$ at $52 \mathrm{kHz}$ is applied to the proof mass, resulting in the amplitude modulation of the motional signal, Fig. 13. Two demodulations, first at the carrier frequency and then at the drive frequency, are used to extract the motional signal from the total EAM pickup signal [19]. Fig. 14 shows the detected rate response of the gyroscope [18] fabricated with a scale factor of $0.43 \mathrm{mV} /(\% / \mathrm{s})$ over an input range of $\pm 250^{\circ} / \mathrm{s}$, confirming suitability of the folded MEMS IMU approach and the fabrication sequence described in Section III.

\section{DISCUSSION AND CONCLUSION}

A new method for creating a chip-scale IMU is described using foldable MEMS structures. A wafer-scale fabrication process is illustrated for manufacturing IMUs with in-situ SOI sensors and feasibility is demonstrated with several challenges identified. Operational accelerometers and gyroscopes are characterized, indicating compatibility of the overall fabrication process. Due to the modularity of SOI fabrication, several other sensors can be incorporated into the folded MEMS design to 


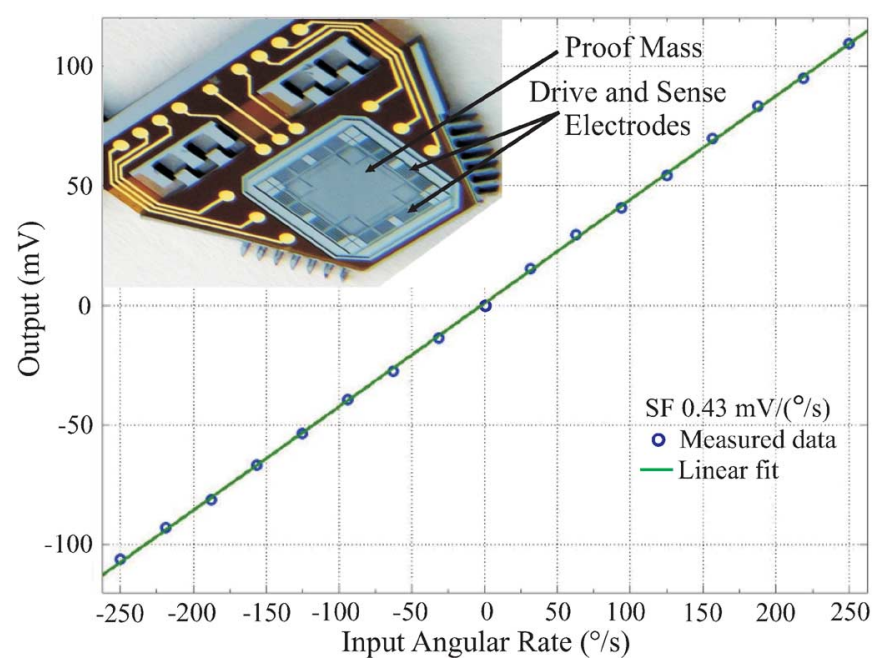

Fig. 14. Measured rate response of a gyroscope integrated on a folded IMU pyramid. Inset: Optical photograph of a fabricated pyramid sidewall with a gyroscope.

create devices such as 3-D microphones, ultrasonic transducers, energy scavengers, and hydrophones.

Pyramidic IMU configurations are investigated. Rigidity of the structures is analyzed, and methods for reinforcement are explored. Alignment stability of epoxy- and solder-bonded IMUs is experimentally investigated under environmental loads including constant acceleration, temperature, thermal cycling, and thermal shock. Reinforcement with epoxy results in modal frequencies occurring above $10 \mathrm{kHz}$, which is suitable for most inertial measurement applications. Bonding with eutectic solder provides much higher rigidity, with the first mode occurring above $50 \mathrm{kHz}$ and therefore will be implemented in harsh vibrational environments. Although a pyramidic shape has been initially explored, other polyhedral form factors can also be implemented. Experimental results confirm feasibility of the proposed folded MEMS IMU approach, and may enable new integrated architectures for other multi-axis dynamic sensors.

\section{ACKNOWLEDGMENT}

The authors would like to acknowledge the University of California, Irvine, Integrated Nanosystems Research Facility (INRF) for assistance with the fabrication of prototypes, D. Senkal for aid in finite-element modeling, and I. Prikhodko for discussions and helping with sensor characterization. The folded IMU structures were designed and characterized at the MicroSystems Laboratory, University of California, Irvine.

\section{REFERENCES}

[1] K. Liu et al., "The development of micro-gyroscope technology," $J$. Micromech. Microeng., vol. 19, no. 11, pp. 1-29, 2009.

[2] M. S. Grewal, L. R. Weill, and A. P. Andrews, Global Position System, Inertial Navigation and Integration. New York: Wiley, 2001.

[3] P. D. Groves, Principles of GNSS, Inertial, and Multisensor Integrated Navigation Systems, 1st ed. Norwood, MA: Artech House, Jan. 2008.

[4] W. Geiger et al., "MEMS IMU for AHRS applications," in Proc. Position, Location and Navigation Symp., May 2008, pp. 225-231.

[5] D. Cardarelli, "An integrated MEMS inertial measurement unit," in Proc. IEEE PLANS, 2002, pp. 314-319.
[6] S. Habibi, S. J. Cooper, J. M. Stauffer, and B. Dutoit, "Gun hard inertial measurement unit based on MEMS capacitive accelerometer and rate sensor," in Proc. PLANS, May 2008, pp. 232-237.

[7] M. Meng, Z. Wu, Y. Yu, Y. Ge, and Y. Ge, "Design and characterization of a six-axis accelerometer," in Proc. IEEE Conf. Robot. Autom., Apr. 2005, pp. 2356-2361.

[8] M. C. Rivers, A. A. Trusov, S. A. Zotov, and A. M. Shkel, "Micro IMU utilizing folded cube approach," in Proc. IMAPS Device Packag. Conf., Mar. 2010, pp. 154-158.

[9] M. C. Rivers, S. A. Zotov, A. A. Trusov, and A. M. Shkel, "Three dimensional folded MEMS technology for multi-axis sensor systems," U.S. Patent, UC Case No. 2010-527.

[10] S. A. Zotov, M. C. Rivers, A. A. Trusov, and A. M. Shkel, "Chipscale IMU using folded-MEMS approach," in Proc. IEEE Sensors, Nov. 2010, pp. 1043-1046.

[11] K. E. Petersen, "Silicon as a mechanical material," Proc. IEEE, vol. 70, no. 5, pp. 420-457, May 1982.

[12] A. K. Brown and Y. Lu, "Performance test results of an integrated GPS/ MEMS inertial navigation package," in Proc. ION GNSS, Sept. 2004, pp. 825-832.

[13] P. G. Savage, "Strapdown system computational elements," in Lecture Series Under the Sponsorship of the Sensors Electronics Technology Panel, St. Petersburg, Russia, May 2004.

[14] S. Sung, J. G. Lee, T. Kang, and J. W. Song, "Development of a tunable resonant accelerometer with self-sustained oscillation loop," in Proc. IEEE Nat. Aerosp. Electron. Conf., 2000, pp. 354-361.

[15] R. Hopkins, J. Miola, W. Sawyer, R. Setterlund, and B. Dow, "The silicon oscillating accelerometer: A high-performance MEMS accelerometer for precision navigation and strategic guidance applications," in Proc. Nat. Tech. Meeting Inst. Navigation, Jan. 2005, pp. 970-979.

[16] S. Sung, C. Kim, J. Park, Y. Lee, and J. Park, "Oscillation amplitudecontrolled resonant accelerometer design using a reference tracking automatic gain control," Int. J. Control, Autom., Syst., pp. 203-210, 2009.

[17] N. Yazdi, F. Ayazi, and K. Najafi, "Micromachined inertial sensors," Proc. IEEE, vol. 86, no. 8, pp. 1640-1659, Aug. 1998.

[18] M. S. Kranz and G. K. Fedder, "Micromechanical vibratory rate gyroscopes fabricated in conventional CMOS," in Proc. Symp. Gyro Technol., Sep. 1997, pp. 3.0-3.8.

[19] A. A. Trusov, A. R. Schofield, and A. M. Shkel, "Performance characterization of a new temperature-robust gain-bandwidth improved MEMS gyroscope operated in air," Sens. Actuators A, vol. 155, no. 1, pp. 16-22, Oct. 2009.

[20] W. P. D'Amico, "Telemetry systems and electric gun projectiles," IEEE Trans. Magn., vol. 37, no. 1, pp. 343-346, Jan. 2001.

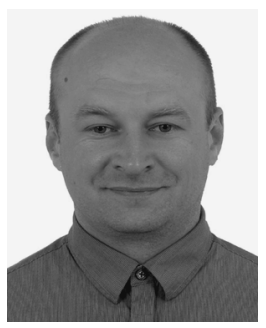

Sergei A. Zotov (M'11) received the M.S. and Ph.D. degrees in mechanical engineering and control systems from the Tula State University, Tula, Russia, in 1999 and 2002, respectively.

$\mathrm{He}$ is currently a Postdoctoral Scientist at the MicroSystems Laboratory, University of California, Irvine, where he is responsible for the design, fabrication, and testing of micromachined devices and systems for inertial navigation. Over the last ten years, his focus has been on MEMS accelerometers and gyroscopes. He holds eight Russian patents and three U.S. patents pending.

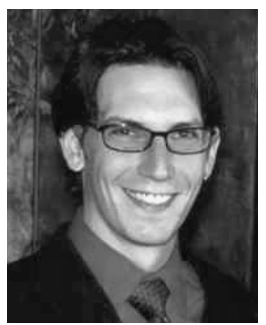

Montgomery C. Rivers (M'11) received the B.S. degree in engineering from Colorado School of Mines, Golden, in 2003 with both electrical and mechanical specialties and an additional minor in computer science, the M.S. degree in mechanical engineering from the University of California, Irvine, in 2008, while conducting research on a folded MEMS three-axis accelerometer. Currently, he is working towards the $\mathrm{Ph} . \mathrm{D}$. degree at the University of California, Irvine, developing chip-scale IMUs using folded MEMS fabrication techniques.

As a product development engineer at Wadsworth Control Systems, he created automated controllers for maintaining environmental conditions for optimal crop growth. He has published two conference papers and submitted two patent disclosures. 


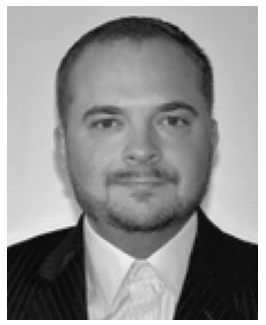

Alexander A. Trusov (M'09) received the B.S. and M.S. degrees in applied mathematics and mechanics from the Moscow State University, Moscow, Russia, in 2004, and the M.S. and Ph.D. degrees in mechanical and aerospace engineering from the University of California, Irvine, in 2006 and 2009, respectively.

He is currently a Project Scientist at the UC Irvine MicroSystems Laboratory, where he serves as a PI and Co-PI on a variety of federally sponsored programs pursuing high-performance inertial microsystems. His research interests include design, modeling, fabrication, and vacuum packaging of micromachined inertial systems, design of characterization experiments, and statistical data processing and analysis. He has published over 40 journal and conference papers on MEMS and inertial sensors, has seven U.S. patents pending, and serves as a reviewer for major journals in the fields of MEMS and sensors.

Dr. Trusov is a member of the American Society of Mechanical Engineers (ASME), and the Institute of Electrical and Electronics Engineers (IEEE). $\mathrm{He}$ is the recipient of an Outstanding Paper Award at the Transducers 2011 Conference for his work on low dissipation silicon MEMS gyroscopes.

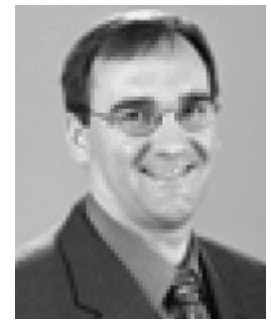

Andrei M. Shkel (SM'08) received the Diploma degree (with excellence) in mechanics and mathematics from the Moscow State University, Moscow, Russia, in 1991, and the Ph.D. degree in mechanical engineering from the University of Wisconsin, Madison, in 1997.

$\mathrm{He}$ is a Program Manager in the Microsystems Technology Office of the Defense Advanced Research Projects Agency (DARPA), Arlington, VA; he is serving in this capacity while on leave from his faculty position as a Professor in the Department of Mechanical and Aerospace Engineering, University of California, Irvine, where he is also the Director of the UCI Microsystems Laboratory. He is the holder of 15 U.S. and international patents. His professional interests are reflected in more than 120 publications.

Dr. Shkel is the recipient of the 2009 Research Award from the IEEE Sensors Council, the 2006 UCI Research Award, the 2005 NSF CAREER Award, and the 2002 George E. Brown, Jr., Award. He is an Editor of the IEEE JOURNAL OF MICROELECTROMECHANICAL SYSTEMS (JMEMS). 